\title{
PM20D1 quantitative trait locus is associated with Alzheimer's disease
}

Jose Vicente Sanchez-Mut ${ }^{1}$, Holger Heyn ${ }^{2,3}$, Bianca Ambrogina Silva ${ }^{1}$, Lucie Dixsaut ${ }^{1}$, Paula Garcia-Esparcia ${ }^{4}$, Enrique Vidal ${ }^{5}$, Sergi Sayols ${ }^{6}$, Liliane Glauser ${ }^{1}$, Ana Monteagudo-Sánchez ${ }^{7}$, Jordi Perez-Tur ${ }^{8}$, Isidre Ferrer ${ }^{4}$, David Monk ${ }^{7}$, Bernard Schneider ${ }^{9}$, Manel Esteller ${ }^{10}$, Johannes Gräff ${ }^{1}$

1 Laboratory of Neuroepigenetics, Brain Mind Institute, Faculty of Life Sciences, Ecole Polytechnique Fédérale de Lausanne, Lausanne, Switzerland

2 CNAG-CRG, Centre for Genomic Regulation (CRG), Barcelona Institute of Science and Technology (BIST), Barcelona, Spain.

3 Universitat Pompeu Fabra (UPF), Barcelona, Spain.

4 Department of Pathology and Experimental Therapeutics, University of Barcelona, IDIBELL, CIBERNED, Hospitalet de Llobregat, Spain.

5 Centre de Regulació Genòmica (CRG), Barcelona Institute for Science and Technology, Barcelona, Spain.

6 Bioinformatics Core Facility, Institute of Molecular Biology, Mainz, Germany.

7 Genomic Imprinting Cancer Group, Cancer Epigenetics and Biology Program, Institut d'Investigació Biomedica de Bellvitge, E-08908 Barcelona, Spain.

8 Institut de Biomedicina de València-CSIC / Ciberned / Instituto de Investigación Sanitaria "La Fe" (València), València, Spain.

9 Neurodegenerative Studies Laboratory, Brain Mind Institute, Faculty of Life Sciences, Ecole Polytechnique Fédérale de Lausanne, Lausanne, Switzerland

10 Cancer Epigenetics Group, Cancer Epigenetics and Biology Program, Institut d'Investigació Biomedica de Bellvitge, Barcelona, Spain.

\section{Corresponding author}

Correspondence to JG: johannes.graeff@epfl.ch 


\begin{abstract}
The chances to develop Alzheimer's disease $(A D)$ result from a combination of genetic and non-genetic risk factors ${ }^{1}$, the latter likely mediated by epigenetic mechanisms ${ }^{2}$. In the past, genome-wide association studies (GWAS) have identified an important number of risk loci associated with $A D$ pathology ${ }^{3}$, but a causal relationship thereof remains difficult to establish. In contrast, locus-specific or epigenome-wide association studies (EWAS) have revealed site-specific epigenetic alterations and thereby provide mechanistic insights for a particular risk gene, but often lack the statistical power of GWAS ${ }^{4}$. Here, combining both approaches, we report a hitherto unidentified association of the Peptidase M20 domaincontaining protein 1 (PM20D1) with AD. We find that PM20D1 is a methylation/expression quantitative trait locus (mQTL/eQTL) coupled to an AD-risk associated haplotype, which displays enhancer-like characteristics and contacts the PM20D1 promoter via a haplotypedependent, CTCF-mediated chromatin loop. Furthermore, PM20D1 is increased following AD-related neurotoxic insults, at symptomatic stages in the APP/PS1 mouse model of AD and in human $A D$ patients, who are carriers of the non-risk haplotype. Importantly, genetically increasing and decreasing the expression of PM20D1 reduces and aggravates AD-related pathologies, respectively. These findings suggest that in a particular genetic background, PM20D1 contributes to neuroprotection against AD.
\end{abstract}




\section{Main}

Over the last decade, the number of epigenomic studies in AD has rapidly increased, which was paralleled by the discovery of an increasing number of epigenetically dysregulated genes ${ }^{5}$. Despite that, only few such genes have been reported by independent studies in humans (i.e., $\mathrm{ANK} 1^{6,7}, \mathrm{SORBS}^{8,9}$ and $\mathrm{HDAC}^{10,11}$ ). Contributing to this discrepancy is the complex heterogeneity of the nervous system, where intra-individual epigenetic as well as inter-individual genetic and epigenetic variability call for more refined and integrative studies ${ }^{5}$. Combining both epigenetic and genetic approaches, it has recently become possible to identify single nucleotide polymorphisms (SNPs) that correlate with alterations in DNA methylation levels - so-called methylation quantitative trait loci (mQTLs) - the importance of which for complex diseases has just started to be recognized ${ }^{4}$. mQTLs have been reported for several neurological disorders including schizophrenia ${ }^{12}$, obsessivecompulsive $^{13}$ and bipolar disorder ${ }^{14}$, but, thus far, not for neurodegenerative diseases such as $A D$.

In two recent EWAS, we previously investigated DNA methylome changes in a panel of neurodegenerative diseases including Parkinson's disease, Lewy body dementia as well as $A D$ at different disease stages and in two major affected brain areas, the hippocampus and the frontal cortex ${ }^{15,16}$. When we re-analyzed these biologically and technically independent datasets focusing exclusively on the comparison between healthy control and advanced stage AD samples, we noticed that only one gene, $P M 20 D 1$, previously described as $\mathrm{mQTL}^{17}$, significantly displayed promoter hypermethylation in AD in both studies (Supplementary Tables 1, 2, Fig. 1a). To confirm this observation, we performed a meta-analysis of publicly available DNA methylation datasets (GSE45775, GSE57361, GSE59685, GSE76105, and GSE80970) and found an enrichment of PM20D1 hypermethylated samples in AD (Fig. 1b,c).

To further investigate the association between the PM20D1 mQTL and AD, we first selected SNPs that significantly correlate with PM20D1 DNA methylation using a recently described mQTL mapping tool ${ }^{12}$ (Supplementary Table 3 ) and examined their relation with AD using a publicly available database from NCBI GWAS studies (phs000168.v1.p1 NIA). We detected a significant allele-dose dependent association with AD for most PM20D1 mQTL-associated SNPs (Supplementary Table 3). The highest significance was observed for SNPs rs708727 and rs960603, which co-segregated as a haplotype in nearly $85 \%$ of cases according to the 1000 Genomes project (Phase III phased haplotypes for CEU population; rs708727 A frequency $0.40 ;$ rs960603 A frequency 0.45$)^{18}$. As the PM20D1 region is poorly represented and in low linkage-disequilibrium with SNPs interrogated in most GWAS arrays, which can differ between different populations ${ }^{19}$, and impinge on SNP imputation accuracy ${ }^{20}$, the rs708727rs960603 haplotype has likely escaped other AD-related GWAS.

Then, we assessed the relationship between the rs708727-rs960603 haplotype, and PM20D1 DNA methylation in human frontal cortex samples. We observed an allele-dependent correlation with PM20D1 promoter methylation (Fig. 1d and e), and further, that PM20D1 
expression was inversely correlated with its promoter methylation (Fig. 1f). Importantly, PM20D1 expression was only detected from rs708727-rs960603 G-C chromosomes in heterozygous samples indicating that PM20D1 is mono-allelically expressed (Fig. 1g). Supporting these observations, rs708727 and rs960603, as well as other mQTL SNPs and the SNPs in linkage-disequilibrium with them (Fig. 1a), have been previously described as PM20D1 eQTLs ${ }^{21}$ (Supplementary Table 3). Of note, although rs708727 lies in the coding region of $S L C 41 A 1$, rs708727 does not change its protein sequence and its expression can be detected from both chromosomes (Fig. 1h). Accordingly, SLC41A1 does not show differences in DNA methylation either (Fig. 1i, j). Importantly, the observed pattern in PM20D1 methylation between $A D$ and control samples cannot be explained by an altered neuron-glia ratio typical for $A D$, since grey and white matter samples from the same samples do not show DNA methylation differences (Supplementary Fig. 1). Taken together, these data point to an association between PM20D1 hypermethylation and $A D$ and show that PM2OD1 methylation and expression are dependent on the rs708727-rs960603 haplotype.

To better understand the molecular relationship between this haplotype and PM2OD1 expression, we immortalized B cells from rs708727-rs960603 carriers (hereafter named unmethylated (UM) and methylated (M) cells for the GG-CC and AA-TT rs708727-rs960603 samples), which show similar SNP-dependent DNA methylation and expression levels of PM20D1 as the human brain samples (Supplementary Fig. 2a, b). Proper control of gene expression often relies on distal cis-regulatory elements that come into physical contact with a gene's promoter region ${ }^{22}$. Using chromatin conformation capture assays (3C), we found that that PM20D1 and its putative regulatory region rs708727-rs960603 interact, but that this interaction was haplotype-dependent i.e., it was weakened in the PM2OD1 transcriptionally silent M cells (Fig. 2a). Next, we used chromatin immunoprecipitation (ChIP) to probe for the binding of CCCTC-binding factor (CTCF), which is responsible for more than $90 \%$ of DNA loops in mammalian genomes ${ }^{23}$. In line with the $3 C$ results, CTCF showed reduced binding to both $3 C$ anchors in $M$ cells (Fig. $\mathbf{2 b}, \mathbf{c}$ ). What is more, we detected increased binding of Methyl CpG Binding Protein 2 (MeCP2) and decreased histone acetylation - two hallmarks of compacted, transcriptionally silent chromatin state in DNAmethylated regions ${ }^{24}$ - in the PM20D1 promoter in M cells (Fig. 2d, e and Supplementary Fig. 2c, d). These epigenetic modifications were functionally important, as treatment with the DNA demethylating agent 5-Azacytidine-dC in combination with the histone deacetylase inhibitor valproic acid not only restored the DNA methylation and histone acetylation changes (Supplementary Fig. 3a-d), but also the chromatin loop (Supplementary Fig. 3e) and PM2OD1 RNA expression (Supplementary Fig. 3f).

Recent evidence suggests that disease-associated SNPs often affect the proper functioning of enhancers ${ }^{25}$. Using the Dragon ENhancers database $(D E N d b)^{26}$, we discovered a predicted enhancer region in proximity to the $3 \mathrm{C} 5^{\prime}$ locus (Supplementary Fig. 4). In line with these in silico data, we found increased levels of the enhancer-enriched epigenetic marks H3K4me1 and $\mathrm{H} 3 \mathrm{~K} 27 \mathrm{ac}^{27}$ in this region, but only in UM cells, which are not methylated at the PM2OD1 
promoter (Fig. 2f, g). Interestingly, this finding is reminiscent of cell lines displaying differential DNA methylation at the PM20D1 promoter, which also show an accumulation for the H3K4me1 and H3K27ac in the enhancer region ${ }^{28}$ (Supplementary Fig. 4). Lastly, in order to test whether this region has transcription-altering properties, we performed luciferase reporter assays and observed a significant increase in luciferase activity in the presence of the predicted enhancer (Fig. 2h). Together, these experiments testify to a haplotypedependent three-dimensional chromatin interaction between the $3 C 5^{\prime}$ locus, with enhancer-like characteristics, and the promoter region of PM20D1, which displays loopdependent differential expression.

Based on the newly described differential expression of PM20D1 according to haplotype and $A D$ risk herein, we next investigated whether PM20D1 might be related to the pathophysiology of $A D$ using a combination of in vitro, mouse in vivo and post mortem human studies. In SH-SY5Y neuroblastoma cells, PM20D1 expression was increased upon treatment with $A D$-related neurotoxic insults such as reactive oxygen species (ROS) and amyloid- $\beta(A \beta)^{1}$ (Fig. 3a). In the APP/PS1 mouse model ${ }^{29}$ of $A D$, which develops AD-related pathologies such as amyloid plaques, astrogliosis and learning deficits starting at seven months of age, all of which increase with age ${ }^{30}, P M 20 D 1$ was higher expressed in the frontal cortex at symptomatic stages as compared to presymptomatic stages and age-matched control mice (Fig. 3b). In human frontal cortex, we analyzed PM20D1 expression after correcting for the rs708727-rs960603 haplotype and found that PM20D1 was increased in non-risk haplotype carriers with $A D$ (Fig. 3c). In line, a meta-analysis of publicly available RNA expression datasets (GSE33000, GSE15222, and GSE36980) shows an increase in PM20D1 expression in rs708727-rs960603 haplotype-corrected samples (Supplementary Fig. 5a). In contrast, PM20D1 expression is decreased in non-haplotype-corrected samples (Supplementary Fig. 5b), which also show an enrichment of PM20D1 repressed samples in AD (Supplementary Fig. 5c). Interestingly, PM20D1 was associated with glia-like cells surrounding neuritic plaques in APP/PS1 mice (Supplementary Fig. 6) and human AD samples as measured by immunohistochemistry (Fig. 3d).

Finally, we genetically manipulated PM20D1 levels using both virus-mediated PM20D1 overexpression and antisense oligonucleotides (ASOs) to mimic elevated and reduced PM20D1 levels, respectively. In vitro, we overexpressed PM2OD1 in SH-SY5Y cells and APP/PS1-derived primary hippocampal cultures using lentiviral constructs and assessed ROSinduced cell death and soluble $A \beta$ levels, respectively. PM20D1 overexpression decreased ROS-induced cell death (Supplementary Fig. 7a) and in primary cultures, where we found PM20D1 to be actively secreted (Supplementary Fig. 7b), its overexpression reduced $A \beta$ levels compared to control-infected cultures (Supplementary Fig. 7c). In vivo, we overexpressed $P M 20 D 1$ using adeno-associated viral vectors (AAVs) stereotaxically delivered to the hippocampus and repressed PM20D1 levels using intracerebroventricular (ICV) injections of ASOs. AAVs and ASOs were injected in asymptomatic 3 month-old APP/PS1 mice and memory performance and amyloid-related pathology assayed 3 months later by 
the novel object recognition tests (NOR) and IHC (Fig. 3e). PM20D1 overexpression (AAV.Mock: $n=8 ; 0.18 \pm 0.05$ vs. AAV.PM20D1: $n=10 ; 544.91 \pm 216.48$, mean \pm SEM for PM20D1 RNA levels; $p<0.05$ by one-tailed student's t-test) reduced amyloid plaque load (Fig. 3f, g) and improved NOR performance (Fig. 3h), while PM20D1 repression (ASO.Scramble: $\mathrm{n}=12 ; 1.00 \pm 0.11$ vs. AAV.PM20D1: $\mathrm{n}=12 ; 0.76 \pm 0.05$, mean \pm SEM for PM20D1 RNA levels; $\mathrm{p}<0.05$ by one-tailed student's t-test) increased amyloid plaque load (Fig. $\mathbf{3} \mathbf{i}, \mathbf{j}$ ) and impaired NOR performance (Fig. 3k). Together, these data describe a so far unknown, protective role for PM20D1 in the progression of AD-related pathologies.

In sum, we provide evidence for a mQTL/eQTL centered on PM20D1 that is implicated in AD. Independent analyses of DNA methylation and RNA expression show a significant enrichment of $P M 2 O D 1$ repression in $A D$, which we find to be linked - via a CTCF-mediated chromatin loop - to an AD-associated haplotype. Functionally, PM20D1 expression is stimulated by neurotoxic insults in vitro and in vivo, while its overexpression reduces cell death, decreases amyloid- $\beta$ levels, and improves cognitive performance. Therefore, elevated levels of PM20D1 might provide a potential cellular defense mechanism for AD non-risk haplotype carriers (Fig. 31). Interestingly, PM20D1 has recently been associated with obesity $^{31-33}$ and diabetes ${ }^{31,33}$, which are themselves risk factors for $A D^{34}$. What is more, PM20D1 has been reported to be differentially methylated in obesity ${ }^{32}$ and multiple sclerosis $^{35}$, and lies within the PARK16 locus on chromosome 1, which has previously been associated with Parkinson's disease ${ }^{36}$. Whether PM20D1 behaves as an epistatic and/or pleiotropic trait across these conditions as well as its precise mode of action now require further investigation.

\section{Acknowledgements}

The work in the laboratory of J.G. is supported by the SYNAPSIS Foundation, the Béatrice Ederer-Weber Stiftung, the Floshield Foundation, and the Alzheimer's Association (NIRG-15363964). The laboratory of D.M. is supported by the Foundation Jérôme Lejeune, Spanish Ministerio de Educación y Competitividad (BFU2014-53093). The laboratory of J.P.-T is supported by the Spanish Ministerio de Economía, Industria y Competitividad and the FEDER programme from the EU (SAF2014-59469-R) and the CIBERNED.

J.V.S.-M is supported by a SYNAPSIS Foundation Advanced PostDocs fellowship and Heidi Seiler-Stiftung foundation. H.H. is a Miguel Servet (CP14/00229) researcher funded by the Spanish Institute of Health Carlos III (ISCIII). B.A.S. is an EMBO long-term fellow (ALTF 16052014, Marie Curie Actions, LTFCOFUND2013, GA-2013-609409). A.M.-S is a recipient of a FPI PhD studentship from MINECO. M.E. is an ICREA Research Professor. J.G. is an MQ fellow and a NARSAD Independent Investigator.

\section{Contributions}

J.V.S.-M, H.H., M.E., and J.G. conceived the project and designed the experiments. J.V.S.-M, B.A.S., L.D., P.G.-E, L.G., A.M.-S, and D.M. performed the experiments. J.V.S.-M, E.V., and S.S. performed the bioinformatic analysis of the data. J.P.-T, I.F., D.M., B.S., D.M., and M.E. 
contributed to the interpretation of the results. The paper was written by J.V.S.-M and J.G. and commented on by all authors.

\section{Competing interests}

A provisional patent application has been filed on the use of PM20D1 methylation and haplotype as biomarkers for Alzheimer's disease (E0103124), with J.V.S.-M, H.H., M.E., and J.G. listed as inventors.

\section{References}

1. Holtzman, D.M., Morris, J.C. \& Goate, A.M. Alzheimer's disease: the challenge of the second century. Science translational medicine 3, 77sr71 (2011).

2. Chouliaras, L., et al. Epigenetic regulation in the pathophysiology of Alzheimer's disease. Progress in neurobiology 90, 498-510 (2010).

3. Scheltens, P., et al. Alzheimer's disease. Lancet (2016).

4. Rakyan, V.K., Down, T.A., Balding, D.J. \& Beck, S. Epigenome-wide association studies for common human diseases. Nature reviews. Genetics 12, 529-541 (2011).

5. Sanchez-Mut, J.V. \& Graff, J. Epigenetic Alterations in Alzheimer's Disease. Frontiers in behavioral neuroscience 9, 347 (2015).

6. Lunnon, K., et al. Methylomic profiling implicates cortical deregulation of ANK1 in Alzheimer's disease. Nature neuroscience 17, 1164-1170 (2014).

7. De Jager, P.L., et al. Alzheimer's disease: early alterations in brain DNA methylation at ANK1, BIN1, RHBDF2 and other loci. Nature neuroscience 17, 1156-1163 (2014).

8. Siegmund, K.D., et al. DNA methylation in the human cerebral cortex is dynamically regulated throughout the life span and involves differentiated neurons. PloS one 2, e895 (2007).

9. Sanchez-Mut, J.V., et al. DNA methylation map of mouse and human brain identifies target genes in Alzheimer's disease. Brain : a journal of neurology 136, 3018-3027 (2013).

10. Graff, J., et al. An epigenetic blockade of cognitive functions in the neurodegenerating brain. Nature 483, 222-226 (2012).

11. D'Addario, C., et al. Transcriptional and epigenetic phenomena in peripheral blood cells of monozygotic twins discordant for alzheimer's disease, a case report. Journal of the neurological sciences 372, 211-216 (2017).

12. Hannon, E., et al. Methylation QTLs in the developing brain and their enrichment in schizophrenia risk loci. Nature neuroscience 19, 48-54 (2016).

13. Stewart, S.E., et al. Genome-wide association study of obsessive-compulsive disorder. Molecular psychiatry 18, 788-798 (2013).

14. Gamazon, E.R., et al. Enrichment of cis-regulatory gene expression SNPs and methylation quantitative trait loci among bipolar disorder susceptibility variants. Molecular psychiatry 18, 340-346 (2013).

15. Sanchez-Mut, J.V., et al. Promoter hypermethylation of the phosphatase DUSP22 mediates PKA-dependent TAU phosphorylation and CREB activation in Alzheimer's disease. Hippocampus 24, 363-368 (2014).

16. Sanchez-Mut, J.V., et al. Human DNA methylomes of neurodegenerative diseases show common epigenomic patterns. Translational psychiatry 6, e718 (2016).

17. Heyn, H., et al. DNA methylation contributes to natural human variation. Genome research 23, 1363-1372 (2013).

18. Genomes Project, C., et al. A global reference for human genetic variation. Nature 526, 68-74 (2015).

19. Li, H., Teo, Y.Y. \& Tan, E.K. Patterns of linkage disequilibrium at PARK16 may explain variances in genetic association studies. Movement disorders : official journal of the Movement Disorder Society 30, 1335-1342 (2015). 
20. Marchini, J. \& Howie, B. Genotype imputation for genome-wide association studies. Nature reviews. Genetics 11, 499-511 (2010).

21. Consortium, G.T. Human genomics. The Genotype-Tissue Expression (GTEx) pilot analysis: multitissue gene regulation in humans. Science 348, 648-660 (2015).

22. Bulger, M. \& Groudine, M. Functional and mechanistic diversity of distal transcription enhancers. Cell 144, 327-339 (2011).

23. Phillips, J.E. \& Corces, V.G. CTCF: master weaver of the genome. Cell 137, 1194-1211 (2009).

24. Suzuki, M., Yamada, T., Kihara-Negishi, F., Sakurai, T. \& Oikawa, T. Direct association between PU.1 and MeCP2 that recruits mSin3A-HDAC complex for PU.1-mediated transcriptional repression. Oncogene 22, 8688-8698 (2003).

25. Hnisz, D., et al. Super-enhancers in the control of cell identity and disease. Cell 155, 934-947 (2013).

26. Ashoor, H., Kleftogiannis, D., Radovanovic, A. \& Bajic, V.B. DENdb: database of integrated human enhancers. Database : the journal of biological databases and curation 2015(2015).

27. Smith, E. \& Shilatifard, A. Enhancer biology and enhanceropathies. Nature structural \& molecular biology 21, 210-219 (2014).

28. Creyghton, M.P., et al. Histone H3K27ac separates active from poised enhancers and predicts developmental state. Proceedings of the National Academy of Sciences of the United States of America 107, 21931-21936 (2010).

29. Borchelt, D.R., et al. Accelerated amyloid deposition in the brains of transgenic mice coexpressing mutant presenilin 1 and amyloid precursor proteins. Neuron 19, 939-945 (1997).

30. Jankowsky, J.L., et al. Mutant presenilins specifically elevate the levels of the 42 residue betaamyloid peptide in vivo: evidence for augmentation of a 42-specific gamma secretase. Human molecular genetics 13, 159-170 (2004).

31. Long, J.Z., et al. The Secreted Enzyme PM20D1 Regulates Lipidated Amino Acid Uncouplers of Mitochondria. Cell 166, 424-435 (2016).

32. Feinberg, A.P., et al. Personalized epigenomic signatures that are stable over time and covary with body mass index. Sci Transl Med 2, 49ra67 (2010).

33. Larrick, J.W., Larrick, J.W. \& Mendelsohn, A.R. Uncoupling Mitochondrial Respiration for Diabesity. Rejuvenation research 19, 337-340 (2016).

34. Kivipelto, M. \& Mangialasche, F. Alzheimer disease: To what extent can Alzheimer disease be prevented? Nature reviews. Neurology 10, 552-553 (2014).

35. Maltby, V.E., et al. Differential methylation at MHC in CD4(+) T cells is associated with multiple sclerosis independently of HLA-DRB1. Clinical epigenetics 9, 71 (2017).

36. Simon-Sanchez, J., et al. Genome-wide association study reveals genetic risk underlying Parkinson's disease. Nature genetics 41, 1308-1312 (2009).

\section{Figures}

Figure 1. Overview of the $P M 20 D 1$ locus and its relation to $A D$ in human frontal cortex. (a) The region comprises several genes (blue arrows) and is in partial linkage-disequilibrium (HapMap CEU Recombination map is indicated below the genes). PM20D1 mQTLs reflected in table 1 are indicated in black, of which the mQTLs most strongly associated with AD in bold magenta (rs708727) and bold red (rs960603). The top ten eQTLs in linkage disequilibrium with rs708727 (magenta) and rs960603 (red) obtained from GTEX database (GTEX consortium, 2015 Science, neuronal origin tissues - Hippocampus \& Tibial Nerve) are also indicated. The differentially methylated region (DMR) on PM20D1 promoter is represented in magenta. (b) Left: Representative heatmap (GSE80970) showing the presence 
of three main methylation groups in - publically available datasets from control and AD IV-VI brain samples using two independent CpG probes - cg1165913 (CpG1) and cg14893161 (CpG2) - located in the PM20D1 promoter. Magenta and green colours indicate high and low levels of DNA methylation in human frontal cortex samples, respectively. Right: Centroidbased clustering (K-means) show three main groups - unmethylated (UM), hemimethylated $(\mathrm{HM})$, and methylated (M) samples - with a higher prevalence of AD samples (black line) in the methylated groups then control samples (CONT, grey line). (c) Cumulative $M$ haplotypes distribution of (b) among control and AD groups ( $n=734$ haplotypes; $p$-val=0.0083; two-sided Chi-square test). ${ }^{* *} p<0.01$. (d) rs708727 correlates with the levels of PM20D1 DNA methylation in human frontal cortex measured by pyrosequencing ( $n=38$ biologically independent human brain samples; $p$-val<0.0001; Pearson correlation). Data are presented as box plots with minimum, first quartile, median, third quartile, and maximum. Single values are represented by dots. (e) rs960603 correlates with the levels of PM20D1 DNA methylation in human frontal cortex measured by pyrosequencing ( $n=34$ biologically independent human brain samples; $p$-val= 0.0008; Pearson correlation). Data are presented as box plots with minimum, first quartile, median, third quartile, and maximum. Single values are represented by dots. (f) PM20D1 RNA expression is inversely correlated with PM20D1 promoter DNA in human frontal cortex samples as measured by quantitative RealTime PCR and pyrosequencing, respectively ( $n=36$ biologically independent human brain samples; $p$-val=0.0005; Pearson correlation). Data are presented as box plots with minimum, first quartile, median, third quartile, and maximum. Single values are represented by dots. (g) rs1361754 is located in the coding region of PM20D1 and is in linkage-disequilibrium with rs708727. Retrotranscription PCR Sanger sequencing of rs1361754 heterozygous samples detects rs1361754 (T) but not rs1361754 (C) RNA transcripts indicating that only the rs708727 (G) chromosome is active. (h) rs708727 is located in the coding region of SLC41A1. Retrotranscription PCR Sanger sequencing of rs708727 heterozygous samples detects both alleles in RNA transcripts indicating that both chromosomes are active. (i) Representative whole genome bisulfite sequencing analysis of DNA methylation in one GG (UM: GSM1173772) and one GA (HM: HM GSM1380998) rs707827 human frontal cortex sample. Differences in DNA methylation are restricted to the DMR region detected in PM20D1 promoter. Dots represents single sequencing reads. Methylation average is represented by smoothed lines, and 95\% confidence intervals by shadows. (j) Locus-specific bisulfite sequencing of PM20D1 region confirming the whole genome bisulfite sequencing and pyrosequencing data.

Figure 2. Long-range chromatin interaction of the rs708727-PM20D1 locus. (a) Genes (blue arrows), PM20D1 DMR (magenta box), and CTCF binding sites from UCSC ENCODE tracks (dark grey boxes) are represented. Long-range interactions between CTCF positive regions have been assayed by chromosome conformation capture assays (light grey boxes) with different primer combinations (black arrows). The graph represents the measured relative interaction frequencies between regions (anchor/HindIII sites) as a function of the genomic distances (in kb) and methylation profile. Ticks in X axis indicate HindIII target sites. PM20D1- 
related mQTLs' relative position is also indicated ( $n=6$ independent experiments; $p$ val=0.0001; two-sided Student's t-test). (b) Chromatin Immunoprecipitation assays (ChIP) showing higher binding of CTCF in UM samples on the $3 C 5^{\prime}$ locus ( $n=4$ independent experiments; $\mathrm{p}$-val= 0.0143; one-sided Student's t-test). (c) ChIP showing higher binding of CTCF in UM samples on the PM20D1 promoter region ( $n=3$ independent experiments; $p$-val= 0.0002; one-sided Student's t-test). (d) ChIP showing higher binding of MeCP2 in M samples on the PM20D1 promoter region ( $n=3$ independent experiments; $p$-val=0.0062; one-sided Student's t-test). (e) ChIP showing that PM20D1 promoter region of M samples is depleted of histone 3 lysine 9 acetylation (H3K9ac) ( $n=3$ independent experiments; $p$-val= 0.0097; one-sided Student's t-test). (f) ChIP showing that H3K4me1 is enriched in UM samples in the 3C 5' locus ( $n=4$ independent experiments; $p$-val=0.0037; one-sided Student's t-test). (g) ChIP showing that H3K27ac is enriched in UM samples in the $3 C 5^{\prime}$ locus ( $n=4$ independent experiments; $p$-val $=0.0081$; one-sided Student's t-test). (h) DENdb predicted enhancer region capacity has been assayed by cloning the region into a pGL4 promoter plasmid and measuring luciferase activity ( $n=8$ independent experiments; $p$-val< 0.0001 ; one-sided Student's t-test). 3C, ChIP and luciferase reporter assay data are presented as means \pm SEM $(a-h)$. Single values are represented by dots $(b-h) .{ }^{*} p<0.05,{ }^{* *} p<0.01,{ }^{* * *} p<0.001$ and $* * * * p<0.0001$.

Figure 3. Functional relevance of $P M 20 D 1$ in $A D$. (a) AD-related stressors such as reactive oxygen species produced by $\mathrm{H}_{2} \mathrm{O}_{2}$ and $A \beta$ treatments increase PM2OD1 RNA expression in $\mathrm{SH}-\mathrm{SY} 5 Y$ cells. Data are presented as means $+\mathrm{SEM}\left(\mathrm{H}_{2} \mathrm{O}_{2} \mathrm{n}=5\right.$ independent experiments; $\mathrm{p}$ val=0.0274; $A \beta n=6$ independent experiments; $p$-val=0.0086; two-sided Student's t-test). (b) $P M 20 D 1$ expression is increased in APP/PS1 mice at the symptomatic stages of pathology. $\mathrm{n}=5$ per genotype and age, data are presented as means + SEM ( $\mathrm{n}=5$ biologically independent mice brain samples; $p$-val=0.0481; one-sided Student's t-test). (c) Haplotypecorrected PM2OD1 RNA levels showing an upregulation of PM2OD1 expression in frontal cortex of human AD samples. Data represent means + SEM of the levels of PM2OD1 RNA expression according to the number of rs708727 G chromosomes of 16 control and $19 \mathrm{AD}$ samples ( $p$-val=0.0148 one-sided Student's t-test). (d) Representative IHC image showing PM20D1 localized in the cytoplasm of neurons and glial cells of AD Braak Stage $V$ samples, with glial cells in the vicinity of $\beta$-amyloid plaque cores showing the strongest immunoreactivity ( $n=10$ biologically independent AD Braak Stage $V$ samples; two sections frontal cortex and hippocampus per case). (e) Timeline and schematic of the genetic manipulations of PM20D1 levels using adeno-associated viral vectors (AAVs) and antisense oligonucleotides (ASO). (f) Representative methoxy-X04 IHC amyloid plaque staining images of AAV.Mock and AAV.PM20D1 injected mice. Three slides per mouse and five mouse per group have been analyzed. (g) Amyloid plaque quantification of (f). $n=5$ per group, data are presented as means + SEM ( $p$-val=0.0123; one-sided Student's t-test). (h) Novel object recognition (NOR) performance of AAV.Mock and AAV.PM20D1 injected mice. Data are presented as box plots with minimum, first quartile, median, third quartile, and maximum of 8 AAV.Mock and 10 AAV.PM20D1 mice ( $p$-val=0.0080; one-sided Student's t-test). (i) Representative methoxy-X04 IHC amyloid plaque staining images of ASO.Scramble and 
ASO.PM20D1 injected mice. Three slides per mouse and five mouse per group have been analyzed (j) Amyloid plaque quantification of (i). $n=5$ per group, data are presented as means + SEM ( $p$-val=0.0296; one-sided Student's t-test). (k) Novel object recognition (NOR) performance of ASO.Scramble and ASO.PM20D1 injected mice. Data are presented as box plots with minimum, first quartile, median, third quartile, and maximum of 12 AAV.Mock and 12 AAV.PM20D1 mice ( $p$-val=0.0167; one-sided Student's t-test). Single values are represented by dots (a-c, g-k). (I) Model for PM20D1 function in AD. Left part: Methylated PM20D1 carriers (shown in magenta) are more frequent among people at risk for Alzheimer's disease (AD) (lower panel) than in healthy control subjects (upper panel), where PM20D1 is unmethylated. Right part: In samples with unmethylated PM20D1 (white open circles representing non-methylated CpG sites, upper part), an enhancer region (depicted by the nucleosome) 60kb downstream of PM20D1 physically interacts with PM20D1 promoter via CTCF-binding (grey ovals), and favors PM20D1 transcription. In the presence of ADrelated stress (red lightning bolt) such as the presence of reactive oxygen species (ROS) and $A \beta, P M 20 D 1$ expression is enhanced, found to surround amyloid plaques, to reduce ROSinduced cell death and $A \beta$ levels, and to prevent memory impairment. In contrast, in samples with hypermethylated PM20D1 (black filled circles representing methylated CpG sites, lower part), the promoter region of PM20D1 is not contacted by the enhancer region, PM20D1 transcription does not occur and there is no protective effect against $A \beta .{ }^{*} p<0.05$ and ${ }^{* *} p<0.01$. Scale bar $(d)=25 \mu \mathrm{m},(\mathrm{f}, \mathrm{i})=1000 \mu \mathrm{m}$. 


\section{Material and Methods}

\section{EWAS data}

EWAS public data was obtained from NCBI GEO database (GSE45775 and GSE57361) and analyzed using R software (http://www.R-project.org) as previously described ${ }^{15,16}$. Briefly, GSE45775 Infinium HumanMethylation27 BeadArray 27K (Illumina) data was quantile normalized using lumi package and analyzed with genefilter package (bioconductor). Differentially methylated regions (DMRs) were defined by two or more probes consistently reporting changes of DNA methylation. GSE57361 whole-genome bisulfite sequencing data was aligned using of Bismark software ${ }^{37}$ and analyzed with SAMtools ${ }^{38}$, bedtools ${ }^{39}$, Tabix ${ }^{40}$, and ggplot2 packages. DMRs were identified by seeking regions with more than five consecutive CpG sites consistently located outside the $95 \%$ confidence interval of the smoothed methylation profile. For the meta-analysis, available NCBI GEO DNA methylation data from control and AD brain samples (GSE45775 ${ }^{15}, \mathrm{GSE} 7361^{16}, \mathrm{GSE}^{16} 9685^{6}$, GSE76105 ${ }^{41}$, and GSE80970 ${ }^{27}$ ) was further investigated. Samples were stratified in three segments according to the corresponding methylated (M), hemimethylated (HM), and nonmethylated (M) samples - using the centroid-based clustering (K-means) method - for each of the studies independently. Then, the cumulative distribution of the segments between control and $A D$ groups was analyzed by Chi-square test. Second order and two neighbors smoothing was used for showing the differential distribution of methylated samples between controls and $A D$ groups. Source codes are available upon request.

\section{GWAS data}

GWAS public data was obtained from NCBI GEO database (phs000168.v1.p1 NIA - Late Onset $A D$ and National Cell Repository for AD Family Study consisting of 607 families - 1,516 affected, 1,306 unaffected). The GWAS NIALOAD dataset was selected based on the high frequency of mehylated SNPs in the population and the direct interrogation of PM20D1 mQTL SNPs without imputation. Selected mQTL SNPs were extracted using PLIN v1.07 software ${ }^{42}$ and the relation with $A D$ by fitting a logistic regression via Generalized Estimation Equation (GEE) in a family data under additive genetic model with phenotype as outcome ${ }^{43}$. Hardy-Weinberg equilibrium and allelic test was performed as previously reported ${ }^{44}$. Linkage-disequilibrium plot was obtained from UCSC track for HapMap Release 24 CEU recombination map and HapMap Linkage Disequilibrium - Phase II - from Phased Genotypes. Haplotypes were obtained from Phase III 1000 Genomes CEU population (http://phase3browser.1000genomes.org). Source codes are available upon request.

\section{Brain samples}

Post-mortem tissues were obtained from the IDIBELL Biobank, which is part of the eBrainNet Europe Bank (http://www.brainnet-europe.org/) 'Network of Excellence' funded by the European Commission in the 6th Framework Program 'Life Science' (LSHM-CT-2004-503039). Informed consent was obtained from all participants. The collection of all samples is conform 
to the relevant regulations, ethical considerations and legislation as defined by the European Union. Samples were dissected and characterized for Braak stage ${ }^{45}$ before further examination. DNA and RNA from grey matter samples of frontal cortex were extracted for subsequent experiments. Only samples with RIN>6.5 according to the RNA quality test on Agilent's 2100 bioanalyzer were included in the study. These filtered samples were DNA and RNA from grey matter of frontal cortex (Brodmann area 9) of 22 controls (Braak 0-II; 32\% female; age $64 \pm 3$ years, mean \pm SEM) and 23 Alzheimer's disease (Braak V-VI; 43\% female; age $77 \pm 2$ years, mean \pm SEM) samples matched for age and gender.

\section{Cell culture}

Immortalized B cells ${ }^{46}$ were cultured in RPMI 1640 medium supplemented with $10 \%$ FBS, 100 $\mu / \mathrm{ml}$ penicillin and $100 \mathrm{mg} / \mathrm{ml}$ streptomycin at $37^{\circ} \mathrm{C}$ in a humidified atmosphere of $5 \% \mathrm{CO}_{2}$. Three independent clones per genotype were used. SH-SY5Y neuroblastoma cells (ATCC) were cultured in DMEM medium supplemented with $20 \%$ FBS, $100 \mu / \mathrm{ml}$ penicillin and 100 $\mathrm{mg} / \mathrm{ml}$ streptomycin at $37^{\circ} \mathrm{C}$ in a humidified atmosphere of $5 \% \mathrm{CO}_{2}$. SH-SY5Y cells were treated with with $0.2 \%$ Hydrogen peroxide (Merck) and with synthetic amyloid- $\beta$ (1-42)derived diffusible ligands (ADDLs) (abcam) prepared as previously described ${ }^{47}$ during 6 and $24 \mathrm{~h}$ respectively.

\section{Mice}

APP/PS1 mice were maintained under standard animal housing conditions in a normal $12 \mathrm{~h}$ light-dark cycle with ad libitum access to food and water. All animal procedures were conducted according to EPFL's and Switzerland's guidelines on animal welfare (cantonal animal experimentation authorization numbers VD2875 and VD3169).

Stereotaxic injections were performed using a glass pipette (intraMARK, 10-20 $\mu \mathrm{m}$ tip diameter, Blaubrand, injection flow: $0.1 \mu \mathrm{l} / \mathrm{min}$ ) connected to a syringe and a stereotaxic micromanipulator (Kopf Instruments) in deeply anesthetized 3 month-old APP/PS1 male mice. After injection the capillary was left at the injection site for 5 min before slow withdrawal at $0.01 \mathrm{~mm} / \mathrm{s}$. Deep anesthesia was induced by subcutaneous injection of a mixture of Fentanyl ( $0.05 \mathrm{mg} / \mathrm{kg}$, Sintetica), Midazolam ( $5 \mathrm{mg} / \mathrm{kg}$, Actavis), Medetomidin (0.5 $\mathrm{mg} / \mathrm{kg}$, Orion Pharma). At the end of the surgical procedure an anesthesia reversal mix containing Naloxon (1.2 mg/kg, Swissmedic), Flumazenil $(0.5 \mathrm{mg} / \mathrm{kg}$, Actavis) and Atipamezol ( $2.5 \mathrm{mg} / \mathrm{kg}$, Orion Pharma) was injected subcutaneously and animals were kept warm for an additional $2 \mathrm{~h}$ and were administered paracetamol $(500 \mathrm{mg} / 250 \mathrm{~mL})$ in the drinking water for 5 days.

AAV (Serotype 9) vectors encoding human PM20D1 (AAV.PM20D1) or mock (AAV.Mock) were bilaterally injected the AAV-containing saline solution $(0.5 \mu \mathrm{l}$ per side) in the dorsal hippocampus (posterior: - $2.00 \mathrm{~mm}$; depth: - $2.25 \mathrm{~mm}$; lateral: $\pm 1.50 \mathrm{~mm}$; coordinates adapted from the Paxinos Atlas).

ASO LNA GapmeR (Exiqon) 5'-CGgAGACCGTAGCGAA-3' (ASO.PM20D1) and ASO LNA GampeR 5'-AACACGTCTATACGC-3' (ASO.Scramble) were used to repress PM20D1 in APP/PS1 
mice. We unilaterally injected $1 \mu \mathrm{l}$ of the ASO-containing PBS Ca ${ }^{2+} / \mathrm{Mg}^{2+}$ free solution $(2.5 \mu \mathrm{g}$ of ASO per animal) in the right ventricle (posterior: - $0.3 \mathrm{~mm}$; depth: - $2.5 \mathrm{~mm}$; lateral: 0.9 $\mathrm{mm}$; coordinates adapted from the Paxinos Atlas). For both AAV and ASO treatments, all animals were randomly assigned to the treatment groups and included in the analysis, except for animals with clearly misplaced injection sites as determined by reporter gene fluorescence and/or needle track localization.

Three months after surgeries, mice were handled $5 \mathrm{~min} /$ day during five consecutive days the week before the novel object recognition test. On test day one, mice were habituated for 10 $\min$ to the open arena. On the second day, mice were placed for $10 \mathrm{~min}$ in the arena with two identical objects, and the time exploring each object was automatically recorded (Ethovision, Noldus). On the third day, one of the two familiar objects was replaced by a novel object. The time exploring each of the objects was recorded and an object recognition index was calculated as the time spent exploring the novel object divided by the total time spent exploring the two objects $\left(\mathrm{RI}=\right.$ time $_{\text {novel }} /$ time $_{\text {novel }}+$ time $\left._{\text {familiar object }}\right)$.

\section{DNA methylation}

DNA was isolated from post-mortem human brain samples by phenol-chloroform extraction and bisulphite converted using the EZ DNA methylation kit (Zymo Research) according to published modifications ${ }^{48}$. Primers for bisulfite cloning/sequencing were designed using the Methyl Primer Express, version 1.0 (Applied Biosystems), and PCR products cloned in the pGEMT-easy sequencing vector (Promega) and Sanger-sequenced as previously described ${ }^{49}$. Primers for pyrosequencing were designed using PyroMark assay design program, version 2.0.01.15 (Qiagen), and pyrosequencing reactions analyzed using PyroMark Q24 System version 2.0.6 (Qiagen) following the manufacturer's instructions. Primers for bisulfite cloning/sequencing and pyrosequencing are listed in Supplementary Table 4.

\section{RNA expression}

Total RNA purification and DNase treatment were performed using TRIzol (Invitrogen) and the Turbo DNA-Free kit (Ambion). RNA was reverse-transcribed using the Thermoscript RTPCR system (Invitrogen), and PCR run using StepOnePlus Real-Time PCR System (Applied Biosystems) and SYBR Green PCR MasterMaster Mix (Applied Biosystems). PCR efficiencies were calculated using standard dilutions and the LinReg software ${ }^{50}$ and three housekeeping genes (GUSB, RPL38 and TBP) were used for normalizing PCR signals. Primers for ChIP are listed in Supplementary Table 4. For the meta-analysis, available NCBI GEO RNA expression data from frontal cortex brain samples of control and AD $\left(\mathrm{GSE} 33000^{51}, \mathrm{GSE} 15222^{52}\right.$, and GSE36980 ${ }^{53}$ ) was further investigated. Samples were stratified in three segments according to the corresponding levels of expression using the centroid-based clustering (K-means) method for each of the studies independently. Then, the aggregated distribution of the segments between control and AD groups was analyzed by Chi-square test. Z-scores were used for comparing overall and stratified levels of expression between controls and AD. RNA 
expression datasets were selected based on the number of samples and the alienated frequency of observed/expected frequencies of alleles.

\section{Chromosome Conformation Capture}

Chromatin conformation capture protocol (3C) was carried out as described elsewhere ${ }^{54}$. In brief, cells were crosslinked 5 min with formaldehyde $2 \%$, blocked 5 min with Glycine 125 $\mathrm{mM}$, and lysed using a hypotonic buffer solution (Sucrose $0.25 \mathrm{M}, \mathrm{KCl} 25 \mathrm{mM}, \mathrm{MgCl}_{2} 5 \mathrm{mM}$, Tris-HCl 20 mM, pH 7.5). Nuclei were resuspended in CutSmart Buffer (New England Biolabs) and permeabilized with Triton-X100 $0.2 \%$. Chromatin was digested overnight with HindIII (New England Biolabs) and ligated overnight in diluted condition with T4 DNA ligase (New England Biolabs). Crosslinks were then reversed overnight at $65^{\circ} \mathrm{C}$ with $200 \mathrm{mM} \mathrm{NaCl}$ and proteinase $\mathrm{K}$ treated. DNA was phenol-chloroform purified, ethanol precipitated, and resuspended in TE. BAC RRP11-219P13 (CHORI) was used for assaying all potential 3C products and primer PCR efficiencies, and efficiency of proximity ligation and DNA concentration for normalizing $3 C$ signals. Primers for $3 C$ are listed in Supplementary Table 4.

\section{Chromatin immunoprecipitation assay}

ChIP experiments were carried out as described previously ${ }^{55}$. Briefly, cells were crosslinked 10 min with formaldehyde $1 \%$, blocked 5 min with Glycine $125 \mathrm{mM}$, and cytoplasm lysed using cell lysis buffer (HEPES $5 \mathrm{mM}, \mathrm{KCl} 85 \mathrm{mM}, \mathrm{NP} 400.5 \%$, pH 8). Nuclei were lysed with nuclear lysis buffer (Tris-HCl 50 mM, EDTA $10 \mathrm{mM}$, SDS 1\%, pH 8) and sonicated to 300 bp average size with EpiSonic Multi-Functional Bioprocessor 1100 (EpiGentek). A total of 5-20 $\mu \mathrm{g}$ per sample and ChIP reaction was immunoprecipitated overnight at $4^{\circ} \mathrm{C}$ with $\mathrm{H} 3 \mathrm{~K} 4 \mathrm{me} 1$ (abcam), H3K9ac (abcam), H3K14ac (abcam), H3K27ac (abcam), H4K12ac (abcam), CTCF (Millipore), and MeCP2 (Sigma) antibodies in diluted condition. Immunoprecipitated chromatin was then isolated using IgG Dynabeads (Life Sciences), and washed twice with low salt buffer ( $\mathrm{NaCl} 150$ mM, SDS $0.1 \%$, NP-40 1 \%, EDTA 1 mM, Deo-Na $0.5 \%$, Tris-HCl 50 mM, $\mathrm{pH}$ 8), high salt buffer ( $\mathrm{NaCl} 500$ mM, SDS $0.1 \%$, NP-40 1 \%, EDTA 1 mM, Deo-Na $0.5 \%$, Tris$\mathrm{HCl} 50 \mathrm{mM}, \mathrm{pH}$ 8), lithium buffer (LiCl 250 mM, SDS $0.1 \%$, NP-40 1 \%, EDTA 1 mM, Deo-Na $0.5 \%$, Tris- $\mathrm{HCl} 50 \mathrm{mM}, \mathrm{pH}$ 8), and TE buffer (Tris- $\mathrm{HCl} 10 \mathrm{mM}$, EDTA $0.25 \mathrm{mM}, \mathrm{pH} 8$ ). Chromatin complexes were eluted with a solution of $\mathrm{NaHCO}_{3} 0.1 \mathrm{M}$ and SDS $1 \%$, and decrosslinked overnight at $65^{\circ} \mathrm{C}$ with $200 \mathrm{mM} \mathrm{NaCl}$ and proteinase $\mathrm{K}$ (Promega). DNA was phenol-chloroform extracted, ethanol precipitated, and resuspended in $50 \mu \mathrm{l} \mathrm{TE}$. PCR was carried out in triplicate using SYBR Green PCR MasterMaster Mix (Applied Biosystems). Thermocycling conditions were $10 \mathrm{~min}$ at $95^{\circ} \mathrm{C}$, then 50 cycles of $15 \mathrm{~s}$ at $95^{\circ} \mathrm{C}$ and $1 \mathrm{~min}$ at $60{ }^{\circ} \mathrm{C}$. Fluorescent signals were acquired by the StepOnePlus Real-Time PCR System (Applied Biosystems). Values were normalized to total DNA input. Primers for ChIP are listed in Supplementary Table 4.

\section{Cloning}


Human PM20D1 (AK057131.1) was cloned from a library of oligodT cDNA derived from SHSY5Y neuroblastoma cells. rs708727 enhancer and control regions was cloned from immortalized $B$ cells DNA. For PM20D1, forward 5'AAAAGAATTCGCCACCATGGCTCAGCGGTGC-3' and reverse 5'CAAGGGATCCAGATGTCGGGAGGAAGGG-3' primers, containing EcoRI and Notl digestion sites respectively, were used. For the rs708727 enhancer, forward 5'AGTAGGTACCCTTAGCTTAGCCATGTCATAGCCTTC-3' and reverse $3^{\prime}$ CGACGCGTCGATATTGGTTTACTCTCGTATCCTTAAAAAG-3' primers were used, containing KpnI and Mlul digestion sites respectively. For the enhancer control region - a region near to rs708727 enhancer not enriched in active enhancers histone marks - forward 5' CGACGCGTCGGGAATACTCACGGCACTGGT-3' and reverse ${ }^{\prime}$ AGTACTCGAGGGGCCATTTGCTATGTCACT-3', containing Mlul and Xhol digestion sites respectively. Transgenes were amplified with high fidelity Prime STAR Max DNA Polymerase (Takara) according to the manufacturer's instructions. PCR products were cloned in pGEMTeasy (Promega) for amplifying the yield. Inserts were released by EcoRI and Notl, and Kpnl and Mlul (New England Biolabs) digestions and ligated into pLVX-IRES-ZsGreen1vector (Promega) and pGL4-promoter Luciferase Reporter Vector (Promega) respectively. All cloned plasmids were verified by Sanger sequencing.

\section{Luciferase assays}

SH-SY5Y neuroblastoma cells (ATCC) were transfected with pGL4-promoter Luciferase reporter vector (Promega) containing mock or predicted enhancer regions using $\mathrm{X}$ tremeGENE HP DNA transfection reagent (Roche). After two days, cells were lysed and enhancer activity measured using Luciferase Assay System (Promega) according to the manufacturer's instructions using Tecan's Infinite M200 PRO reader.

\section{Cell viability assays}

SH-SY5Y neuroblastoma cells (ATCC) were transfected with pLVX-IRES-ZsGreen1vector (Promega) containing mock or PM20D1 gene using X-tremeGENE HP DNA transfection reagent (Roche). Cells were harvested and seeded in 96 well plates $24 \mathrm{~h}$ later, and treated with $250 \mu \mathrm{M} \mathrm{H}_{2} \mathrm{O}_{2}$ during $24 \mathrm{~h}$ next day. Cell viability was measured using Alamablue ${ }^{\circledR}$ cell viability assay (Invitrogen) according to the manufacturer's instructions using Tecan's Infinite M200 PRO reader.

\section{Lentiviral production}

Lentiviral vectors were produced in HEK-293T cells using a third generation packaging system by calcium phosphate transfection with the following plasmids: pMD2.G $(2.5 \mu \mathrm{g})$, psPAX2 $(7.5 \mu \mathrm{g})$ and pLVX-IRES-ZsGreen1vector containing PM20D1/mock sequence (10 $\mu \mathrm{g})$. After four days, medium was collected and centrifuged in a SW32Ti ultracentrifuge rotor at $19,000 \mathrm{rpm}$ for $90 \mathrm{~min}$ at $4{ }^{\circ} \mathrm{C}$. The pellet was resuspended in $120 \mu \mathrm{l}$ of buffer containing $1 \times$ 
PBS pH 7.4 and $0.5 \%$ BSA for a 50X concentrated virus stock. Viral titer was determined using the HIV-1 p24 antigen ELISA kit (Zeptometrix Corp).

\section{Amyloid-beta assays}

Primary hippocampal neuron-glia co-cultures derived from P0 APP/PS1 mice ${ }^{29}$ were cultured in media consisting of Neurobasal (Invitrogen), B27 supplement (Invitrogen), L-glutamine (Invitrogen) and penicillin/streptomycin (Invitrogen) $(0.2 \mathrm{ml}$ per well) on 96 wells plates $\left(2.5 \times 10^{4}\right.$ cells per well ) coated with Cultrex poly-L-lysine (Trevigen). Cells were infected at DIV5 with $10-40 \times 10^{3}$ (100-400 ng per well) viral particles containing either a GFP (mock) or PM20D1 version of the plasmid. At DIV12 media from 96 wells was collected and measured Using the Amyloid beta 40 ELISA Kit (Novex) according to the manufacturer's instructions.

\section{Amyloid Plaques}

Mouse coronal brain slices $(20 \mu \mathrm{m})$ were mounted on Superfrost Plus slides coated with Vectabond (Thermo Scientific Laboratories). Sections were fixed in $4 \%$ paraformaldehyde for $5 \mathrm{~min}$, washed in PBS, and then stained for 15 minutes with a solution of $10 \mu \mathrm{g} / \mathrm{ml}$ MethoxyX04 (Abcam) in 50\% ethanol. Finally, sections were washed in 50\% ethanol and in water, dried, and coverslipped with Fluoromount-G (southern biotech). Images were acquired with the EVOS FL digital microscope (Thermo Fischer Scientific) and quantified using ImageJ.

\section{Immunohistochemistry}

Immunohistochemical study of PM20D1 was performed in $4 \mu \mathrm{m}$-thick dewaxed paraffin sections of 18 months APP/PS1 mice and AD Braak stage V-VI frontal cortex area 8 of human brains. Tissue sections were boiled in citrate buffer during $20 \mathrm{~min}$ to retrieve antigenicity. Endogenous peroxidases were blocked with peroxidase (Dako) followed by $10 \%$ normal goat serum. The primary antibody incubated overnight at 4 으 $\mathrm{C}$ was the rabbit polyclonal antiPM20D1 (Sigma-Aldrich). Following, the sections were incubated with super sensitive link label IHC Biogenex Kit system at room temperature. The peroxidase reaction was visualized with diaminobenzidine (DAB) and $\mathrm{H}_{2} \mathrm{O}_{2}$. The omission of the primary antibody in the same section was used as a control of the immunostaining; no signal was obtained with the incubation of the secondary antibody only (data not shown). Sections were slightly counterstained with haematoxylin.

\section{Western Blotting}

Primary hippocampal cultures derived from wild-type mice were cultured on $35 \mathrm{~mm}$ well plates $\left(2.5 \times 10^{5}\right.$ cells per well) coated with Cultrex poly-L-lysine (Trevigen). Cells were infected at DIV6 with $10-40 \times 10^{4}$ (1-4 $\mu \mathrm{g}$ per well) viral particles containing either a GFP (mock) or PM20D1 version of the plasmid. At DIV14 cells and media were collected, mixed with Laemmli buffer and concentration normalized using the DCA assay (Bio Rad). Equal amounts of protein (30 $\mu \mathrm{g}$ per lane) were separated by SDS-PAGE (10\%) and transferred onto nitrocellulose membranes (Amersham, GE Healthcare). Ponceau S staining (SigmaAldrich) was used for monitoring protein loading and transference. Non-specific binding was 
blocked by incubation in 5\% non-fat milk in Tris-buffered saline $(100 \mathrm{mM} \mathrm{NaCl}, 10 \mathrm{mM}$ Tris, $\mathrm{pH}$ 7.4) containing $0.2 \%$ Tween (TTBS) for $1 \mathrm{~h}$ at room temperature. Afterwards, membranes were incubated overnight at $4^{\circ} \mathrm{C}$ with the polyclonal rabbit anti-PM20D1 (Sigma-Aldrich) in TTBS with $3 \%$ bovine serum albumin. For cell lysates, protein loading was also monitored using a mouse monoclonal antibody against $\beta$-actin (Sigma-Aldrich). Membranes were then incubated for $1 \mathrm{~h}$ in the appropriate horseradish peroxidase-conjugated secondary antibodies (Dako) and immunocomplexes were revealed by an enhanced chemiluminescence reagent (ECL AdvanceTM, Amersham Biosciences). Densitometric quantification was carried out with Fusion FX software (Vilber).

\section{Statistics}

Statistical analysis was done using R package and Prism 6.0 (GraphPad) as described in the figure legends. All experimental data points were included in the analysis, unless they were statistical outliers as determined by Grubb's outlier calculation's (GraphPad Prism). All in vitro and in vivo experiments were conducted by experimenters blinded to the experimental conditions, and controlled for their normal distribution.

\section{Life Sciences Reporting Summary}

Further information on experimental design is available in the Life Sciences Reporting Summary.

\section{Data availability.}

Data from these experiments are available from the corresponding author upon reasonable request. Genetic, DNA methylation, and RNA expression data are available at Gene Expression Omnibus (Genetics: phs000168.v1.p1; DNA methylation: GSE45775, GSE57361, GSE59685, GSE76105, and GSE80970; RNA expression: GSE33000, GSE15222, and GSE36980). A Life Sciences Reporting Summary is available.

\section{References}

37. Krueger, F. \& Andrews, S.R. Bismark: a flexible aligner and methylation caller for Bisulfite-Seq applications. Bioinformatics 27, 1571-1572 (2011).

38. Li, H., et al. The Sequence Alignment/Map format and SAMtools. Bioinformatics 25, 20782079 (2009).

39. Quinlan, A.R. \& Hall, I.M. BEDTools: a flexible suite of utilities for comparing genomic features. Bioinformatics 26, 841-842 (2010).

40. $\mathrm{Li}, \mathrm{H}$. Tabix: fast retrieval of sequence features from generic TAB-delimited files. Bioinformatics 27, 718-719 (2011).

41. Watson, C.T., et al. Genome-wide DNA methylation profiling in the superior temporal gyrus reveals epigenetic signatures associated with Alzheimer's disease. Genome medicine 8, 5 (2016).

42. Purcell, S., et al. PLINK: a tool set for whole-genome association and population-based linkage analyses. American journal of human genetics 81, 559-575 (2007). 
43. Chen, M.H. \& Yang, Q. GWAF: an R package for genome-wide association analyses with family data. Bioinformatics 26, 580-581 (2010).

44. Guedj, M., Wojcik, J., Della-Chiesa, E., Nuel, G. \& Forner, K. A fast, unbiased and exact allelic test for case-control association studies. Human heredity 61, 210-221 (2006).

45. Braak, H. \& Braak, E. Neuropathological stageing of Alzheimer-related changes. Acta neuropathologica 82, 239-259 (1991).

46. Heyn, H., et al. Distinct DNA methylomes of newborns and centenarians. Proceedings of the National Academy of Sciences of the United States of America 109, 10522-10527 (2012).

47. Klein, W.L. Abeta toxicity in Alzheimer's disease: globular oligomers (ADDLs) as new vaccine and drug targets. Neurochemistry international 41, 345-352 (2002).

48. Bibikova, M., et al. Genome-wide DNA methylation profiling using Infinium(R) assay. Epigenomics 1, 177-200 (2009).

49. Fernandez, A.F., et al. The dynamic DNA methylomes of double-stranded DNA viruses associated with human cancer. Genome research 19, 438-451 (2009).

50. Ruijter, J.M., et al. Amplification efficiency: linking baseline and bias in the analysis of quantitative PCR data. Nucleic acids research 37, e45 (2009).

51. Narayanan, M., et al. Common dysregulation network in the human prefrontal cortex underlies two neurodegenerative diseases. Molecular systems biology 10, 743 (2014).

52. Webster, J.A., et al. Genetic control of human brain transcript expression in Alzheimer disease. American journal of human genetics 84, 445-458 (2009).

53. Hokama, M., et al. Altered expression of diabetes-related genes in Alzheimer's disease brains: the Hisayama study. Cerebral cortex 24, 2476-2488 (2014).

54. Court, F., et al. Long-range chromatin interactions at the mouse Igf2/H19 locus reveal a novel paternally expressed long non-coding RNA. Nucleic acids research 39, 5893-5906 (2011). 
Figura 1

a


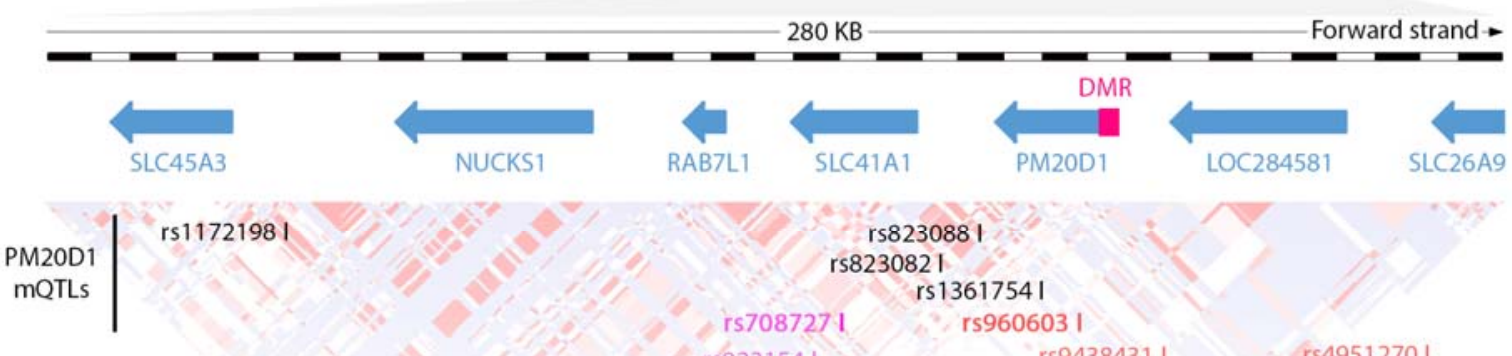

$$
\begin{aligned}
& \text { rs8230881 } \\
& 823082 \text { I } \\
& \text { rs1361754 I }
\end{aligned}
$$

rs708727 I rs960603 I

$\begin{array}{ccc}r 58231411 & \text { rs } 8230741 & \text { rs } 112405741 \quad r s 7537069 \mid \\ r 57087251 & \text { rs } 8230751 & \text { rs6702688 I }\end{array}$

rs8231431 rs94383931 rs7517009| rs4333900

rs708724I rs823080I rs7517201I

rs1772159 I rs9438394I

rs42457211

b

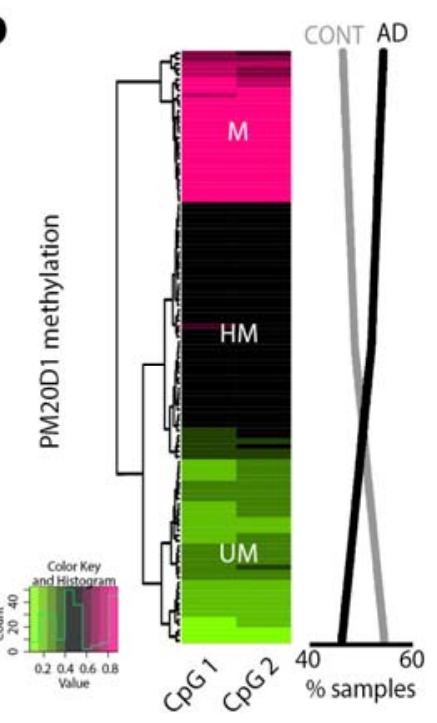

rs708727 eQTLS

\section{d}

Cethylated PM20D1 haplotypes

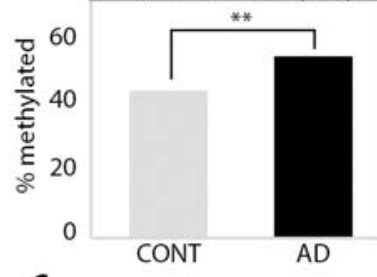

f

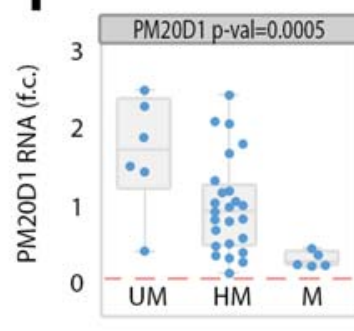

g rs960603 eOTLs

\section{e}
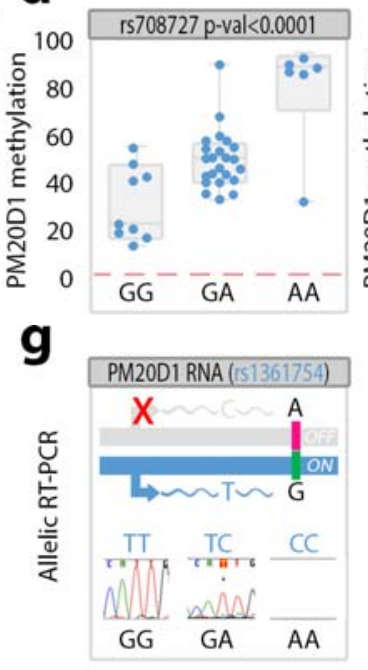

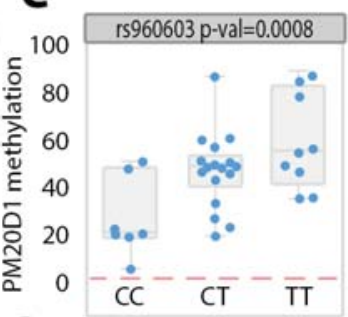

h

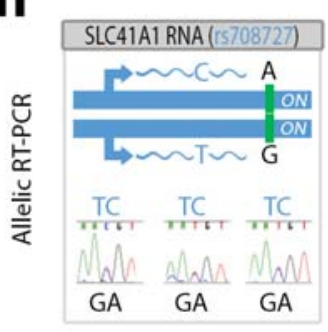

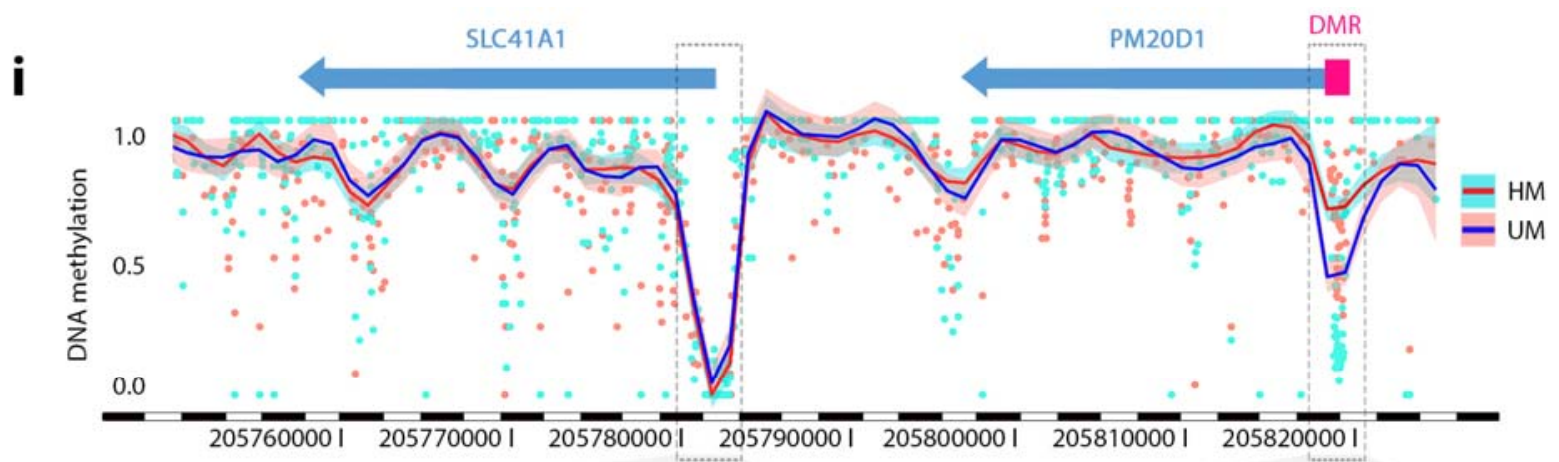

j
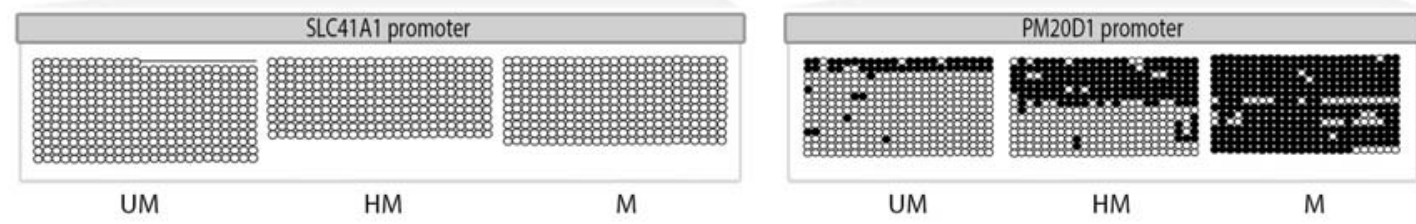
Figura 2

a

$\operatorname{chr} 1$ (q32.1) प w
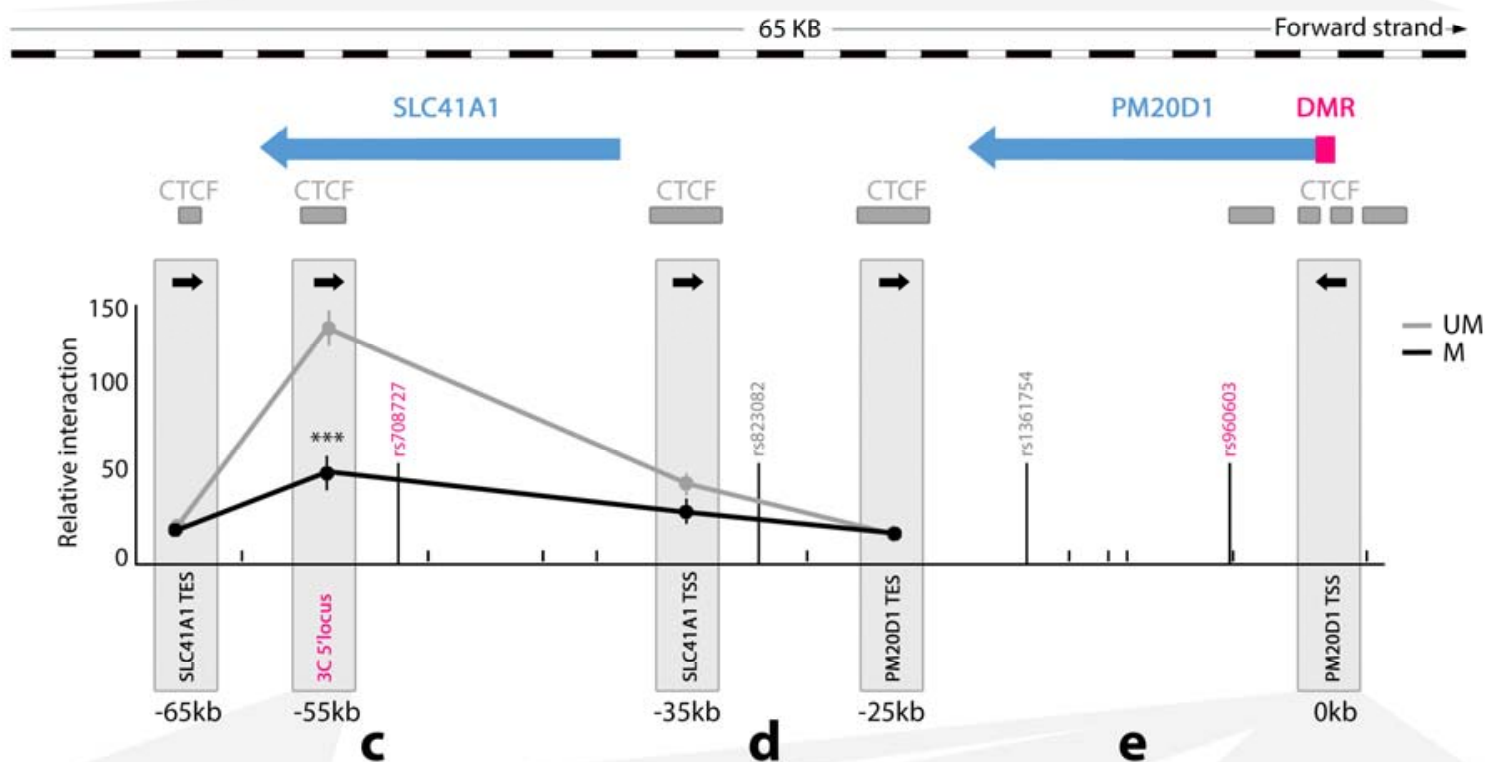

b

$8.0 \quad$ CTCFChIP on rs708727

C.0 CTCF ChIP on PM20D1 prom

12 MeCP2 ChIP on PM20D1 prom

16 H3K9ac ChIP on PM20D1 prom


f

g
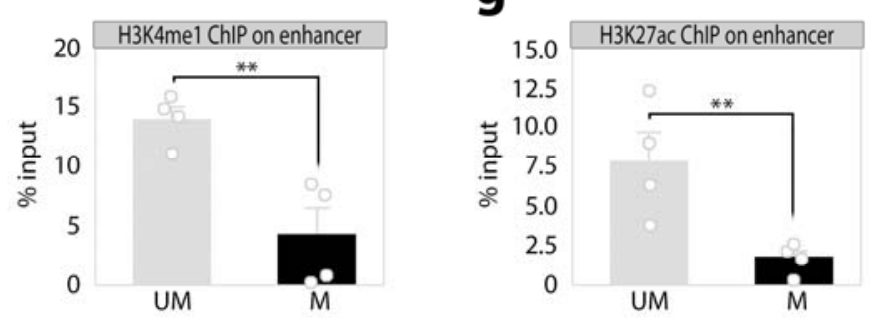

h

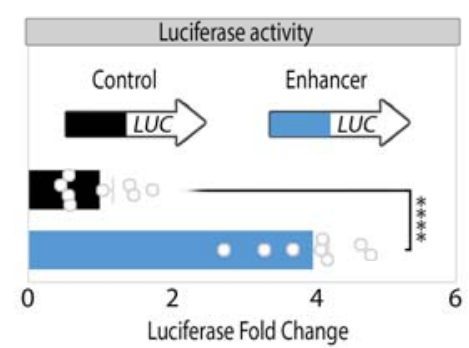




\section{Figura 3}
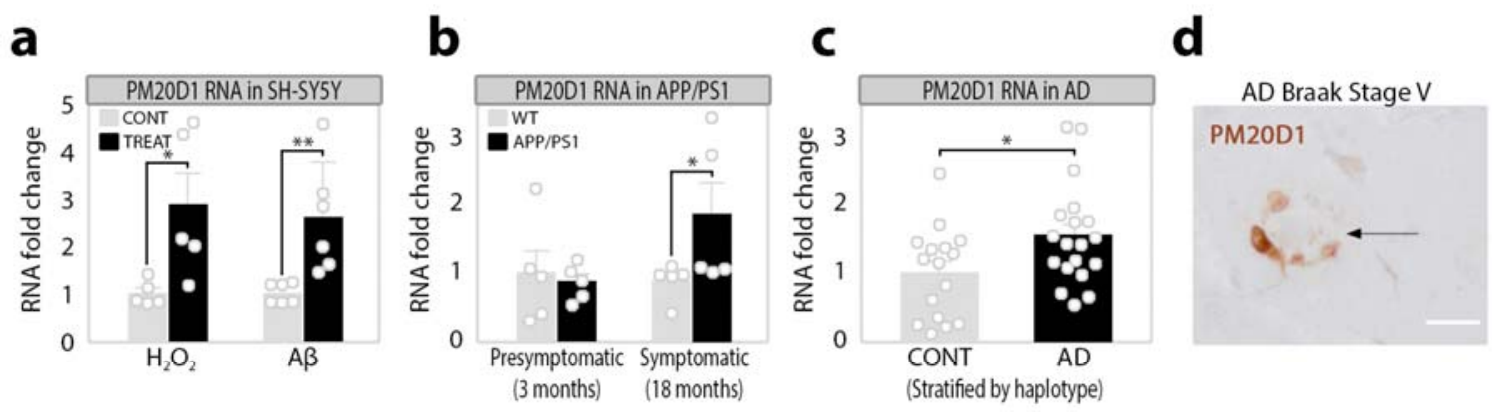

e


ASO-mediated repression of PM20D1

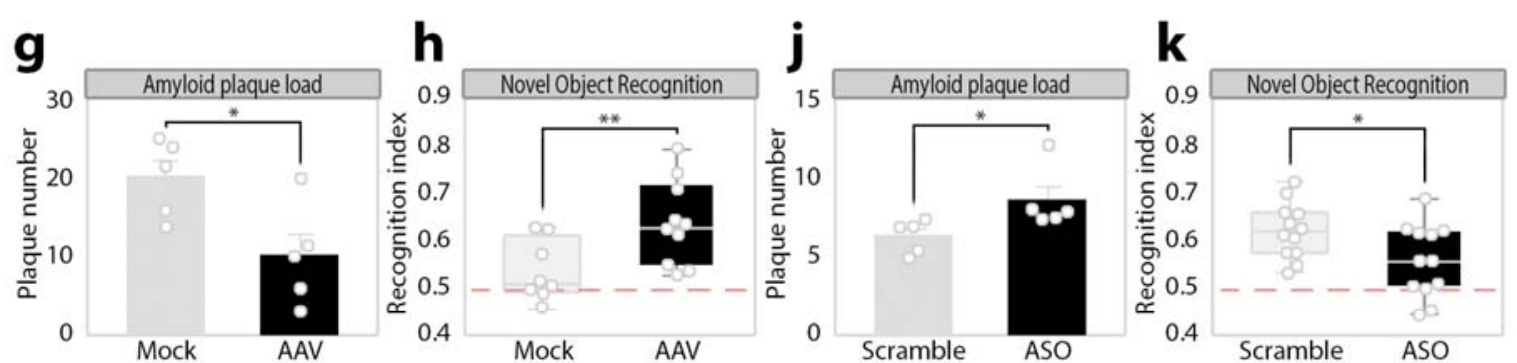

I



PM20D1 chromatin conformation

Outcome
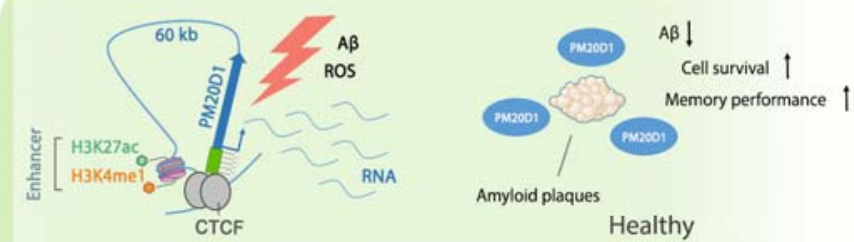

$A \beta \uparrow$

Cell survival !

Memory performance !

Alzheimer 